\title{
Object Location Memory Error in Virtual and Real Environments
}

\author{
Mengxin $\mathrm{Xu}^{*} \quad$ María Murcia-López $^{\dagger} \quad$ Anthony Steed ${ }^{\ddagger}$
}

University College London, UK

\begin{abstract}
We aim to further explore the transfer of spatial knowledge from virtual to real spaces. Based on previous research on spatial memory in immersive virtual reality (VR) we ran a study that looked at the effect of three locomotion techniques (joystick, pointing-andteleporting and walking-in-place) on object location learning and recall. Participants were asked to learn the location of a virtual object in a virtual environment (VE). After a short period of time they were asked to recall the location by placing a real version of the object in the real-world equivalent environment. Results indicate that the average placement error, or distance between original and recalled object location, is approximately $20 \mathrm{~cm}$ for all locomotion technique conditions. This result is similar to the outcome of a previous study on spatial memory in VEs that used real walking. We report this unexpected finding and suggest further work on spatial memory in VR by recommending the replication of this study in different environments and using objects with a wider diversity of properties, including varying sizes and shapes.
\end{abstract}

Index Terms: H.5.1 [Information Interfaces and Presentation]: Multimedia Information Systems-Artificial, Augmented, and Virtual Realities; I.3.7 [Computer Graphics]: Three-Dimensional Graphics and Realism-Virtual Reality

\section{INTRODUCTION}

\subsection{Virtual reality studies on spatial memory}

Several studies have looked at the effect of different technological parameters of virtual reality (VR) that can degrade spatial information transfer from virtual to real environments, including environmental fidelity, field of view and visual complexity [10, 7]. Previous research has also assessed the effect of level of immersion, selfavatar and environmental fidelity on object location memory in virtual environments (VEs) [6]. That study found that HMD learning resulted in statistically significant higher performance than desktop learning and that the use of a virtual body had a negative effect on object location memory. Preliminary inspection of navigation data also indicated that spatial learning strategies are different in systems with varying levels of immersion.

Spatial perception in VEs has also been addressed in a number of experimental tasks based on distance estimation [2, 3, 4, 5]. These have shown that perception of specific spatial characteristics are different in VR and in the real world.

\subsection{Navigating virtual environments}

Locomotion techniques allow users to move around VEs when physical navigation is restricted or not possible [1]. We are interested in understanding if different locomotion techniques have an effect on the ability that users have to acquire spatial knowledge about object locations in immersive VR and transfer this information to the real world. We expect locomotion technique to affect the learning of this spatial information.

In this study we compare the effect of three locomotion techniques (joystick, pointing-and-teleporting and walking-in-place) on

\footnotetext{
*e-mail:mengxin.xu.15@ucl.ac.uk

†e-mail:maria.murcia.13@ucl.ac.uk

‡e-mail:a.steed@ucl.ac.uk
}

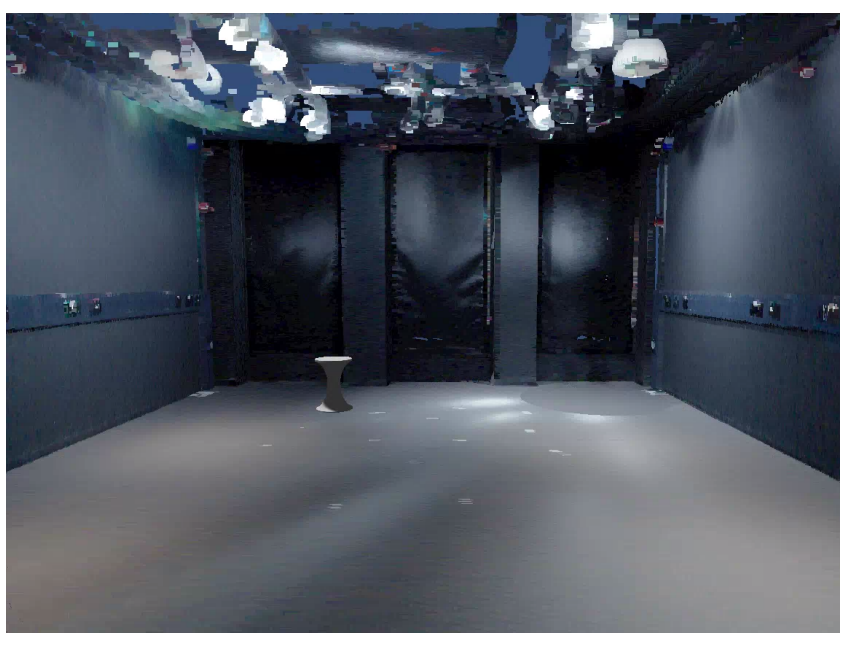

Figure 1: Screenshot of the experimental VE with the virtual object.

object location memory and recall in virtual and real environments. Using one of the three locomotion techniques (each corresponding to one experimental condition), participants could freely navigate the experimental VE to learn the location of a virtual object.

Joystick controller navigation was implemented by allowing participants to use the HTC vive trackpad controller to move. Finger movements on the trackpad were mapped to $\mathrm{X}$ and $\mathrm{Y}$ movement of the user in the virtual space. Participants could reorient by using the trigger and grip buttons, which allowed them to rotate around the vertical axis in clockwise and counterclockwise direction at a constant rotation speed. Under this experimental condition participants were encouraged to use the trigger and grip buttons to reorient but they could also physically rotate.

In the pointing-and-teleporting experimental condition, a ray was projected from the controller to a pointed location on the floor when the participant pressed and held the HTC Vive trackpad. When released, the participant was instantly teleported to the location where the ray intersected the floor plane of the VE. With this locomotion technique the participant could reorient by physically rotating.

The last technique we compared was walking-in-place [8, 9]. An HTC Vive controller was attached to the upper leg of the participant, near the knee. The participant was allowed to walk on a fixed spot and physically rotate to reorient. Changes in controller location due to leg movement were used to simulate changes in speed and position.

\section{Method}

Based on previous research [6], our study examines the effect of locomotion technique on object location memory in VEs. A total of 18 participants ( 9 female, 9 male; average age 26.4 years, $\mathrm{SD}=1.8$ ) were recruited from the student and staff population at University College London. 
Table 1: Mean placement error, standard deviation and standard error (in $\mathrm{m}$ ) for each of the corresponding conditons in the previous study and our study. Note that the column labelled "Real World" corresponds to the experimental condition from the previous study where participants learnt object locations in the real environment with physical versions of the objects. Also note that the column labelled "VE Physical Walking" corresponds to the HMD - No Body - High Detail VE condition condition from the previous study where participants could navigate the VE by physically walking within the limits of the tracking systems.

\begin{tabular}{|l|c|c|c|c|c|}
\cline { 2 - 6 } \multicolumn{1}{c|}{} & \multicolumn{2}{c|}{ Previous study } & \multicolumn{3}{c|}{ Our study } \\
\hline Locomotion Technique & Real World & VE Physical Walking & VE Joystick & VE Pointing-and-Teleporting & VE Walking-in-Place \\
\hline Mean Placement Error & 0.09 & 0.18 & 0.22 & 0.19 & 0.23 \\
\hline Standard Deviation & 0.04 & 0.09 & 0.18 & 0.16 & 0.20 \\
\hline Standard Error & 0.01 & 0.03 & 0.02 & 0.02 & 0.03 \\
\hline
\end{tabular}

Using the virtual reconstruction of the physical environment where the experiment took place, participants were asked to learn the location of a virtual object using the HTC Vive and one of three locomotion techniques: using the HTC Vive controller trackpad as a joystick, pointing-and-teleporting and walking-in-place, as outlined before. Figure 1 contains a screenshot of the experimental VE with the virtual object. After a short period of time participants then completed the recall stage in which they were asked to place a physical version of the object in the real-world equivalent environment. The experimental design of this study was within-subjects, with all participants completing nine trials, three for each locomotion tehcnique. Object location was randomised for each of the trials within a given set of possible locations. The order in which participants experienced the trials was altered so that all orders were equally tested. Participants could freely navigate the environment in all stages of each trial for all experimental conditions and had no time limit to complete the task.

It is important to note that in our study participants learnt and recalled the location of one object in each trial. This is different to the previous study [6], where participants learnt and recalled the location of three identical objects in each trial. The object used in the study was a white Tam Tam plastic stool from Habitat. It was chosen due to its rotational symmetry along the vertical axis, eliminating the question of object orientation.

\section{Results}

We measured placement error, or the Euclidean distance between the original object position and the object position as placed by participants in the recall stage. A repeated measures ANOVA with a Greenhouse-Geisser correction showed that there was no statistically significant effect of locomotion technique on placement error, $F(1.981,104.976)=0.822, p=0.441$.

We then compared our results with results corresponding to the HMD - No Body - High Detail VE condition from a previous study on spatial memory in immersive VR [6], as this experimental condition represents the equivalent experimental setup but using a different locomotion technique (physical walking). Table 1 shows the mean placement error and standard deviations for each of the locomotion techniques. We found that both our study and the previous one show very similar mean placement errors for all locomotion techniques (all of them close to $20 \mathrm{~cm}$ ), except when learning is done in the real world, where the mean placement error is $9 \mathrm{~cm}$.

\section{Conclusion}

We present the results of a study that looks at the effect of three locomotion techniques (joystick, pointing-and-teleporting and walking-in-place) on object location learning and recall in virtual and real environments. Participants were asked to learn the location of a virtual object in a $\mathrm{VE}$ and then place a physical version of the object in the real-world equivalent environment. We measured placement error, or the distance between the original object location in the a VE that was a virtual copy of the real world physical space and the location were each participant placed the physical object in the real world. Our results show no statistically significant effect of locomotion technique on placement error. We also found that the mean placement error for all locomotion techniques highly resembles the mean placement error for a specific experimental condition in a previous experiment [6]. This was a surprise because we expected the locomotion techniques to inhibit spatial learning.

We believe that our results could relate with distance underestimation in VR. Although our task does not explicitly look at distance estimation, the underlying judgments used to complete the task could be the same. Further research is needed including similar studies in different environments as well as varying object number, sizes and shapes. Future research should also explore learning and recall of objects located on vertical planes or walls.

\section{REFERENCES}

[1] D. A. Bowman, D. Koller, and L. F. Hodges. Travel in immersive virtual environments: An evaluation of viewpoint motion control techniques. In Virtual Reality Annual International Symposium, 1997., IEEE 1997, pages 45-52. IEEE, 1997.

[2] J. Campos, H. Nusseck, C. Wallraven, B. Mohler, and H. Bülthoff. Visualization and (mis) perceptions in virtual reality. Tagungsband, 10:10-14, 2007.

[3] V. Interrante, B. Ries, and L. Anderson. Distance perception in immersive virtual environments, revisited. In IEEE Virtual Reality Conference (VR 2006), pages 3-10. IEEE, 2006.

[4] J. M. Knapp and J. M. Loomis. Limited field of view of headmounted displays is not the cause of distance underestimation in virtual environments. Presence: Teleoperators and Virtual Environments, 13(5):572-577, 2004.

[5] J. M. Loomis, J. A. D. Silva, J. W. Philbeck, and S. S. Fukusima. Visual perception of location and distance. Current Directions in Psychological Science, 5(3):72-77, 1996.

[6] M. Murcia-López and A. Steed. The effect of environmental features, self-avatar and immersion on object location memory in virtual environments. Frontiers in ICT, 3:24, 2016.

[7] E. D. Ragan, D. A. Bowman, R. Kopper, C. Stinson, S. Scerbo, and R. P. McMahan. Effects of field of view and visual complexity on virtual reality training effectiveness for a visual scanning task. Visualization and Computer Graphics, IEEE Transactions on, 21(7):794-807, 2015.

[8] J. N. Templeman, P. S. Denbrook, and L. E. Sibert. Virtual locomotion: Walking in place through virtual environments. Presence: teleoperators and virtual environments, 8(6):598-617, 1999.

[9] M. Usoh, K. Arthur, M. C. Whitton, R. Bastos, A. Steed, M. Slater, and F. P. Brooks Jr. Walking - walking-in-place - flying, in virtual environments. In Proceedings of the 26th annual conference on Computer graphics and interactive techniques, pages 359-364. ACM Press/Addison-Wesley Publishing Co., 1999.

[10] D. Waller, E. Hunt, and D. Knapp. The transfer of spatial knowledge in virtual environment training. Presence: Teleoperators and Virtual Environments, 7(2):129-143, 1998. 